\title{
A PARTIAL POSITIVE SOLUTION TO A CONJECTURE OF RICCERI
}

\author{
Francisco J. García-Pacheco - Justin R. Hill
}

\begin{abstract}
In this manuscript we introduce a new class of convex sets called quasi-absolutely convex and show that a Hausdorff locally convex topological vector space satisfies the weak anti-proximinal property if and only if every totally anti-proximinal quasi-absolutely convex subset is not rare. This improves results from [7] and provides a partial positive solution to a Ricceri's Conjectured posed in [9] with many applications to the theory of partial differential equations. We also study the intrinsic structure of totally anti-proximinal convex subsets proving, among other things, that the absolutely convex hull of a linearly bounded totally anti-proximinal convex set must be finitely open. Finally, a new characterization of barrelledness in terms of comparison of norms is provided.
\end{abstract}

\section{Introduction}

In [9] Ricceri establishes the notion of total anti-proximinality and poses a conjecture on the topological structure of such sets.

DeFinition 1.1 (Ricceri, [9]).

(a) Let $E$ be a metric space. A non-empty proper subset $A$ of $E$ is called anti-proximinal exactly when for every element $e \in E \backslash A$ the distance from $e$ to $A, d(e, A)$, is never attained at any $a \in A$.

2010 Mathematics Subject Classification. Primary: 15A03, 46A55; Secondary: 46B20. Key words and phrases. Anti-proximinal, barrelled, Ricceri. 
(b) Let $E$ be a vector space. A non-empty proper subset $A$ of $E$ is called totally anti-proximinal exactly when $A$ is anti-proximinal for every norm on $E$.

(c) A Hausdorff locally convex topological vector space $E$ is said to have the anti-proximinal property if every totally anti-proximinal convex subset is not rare.

(d) A Hausdorff locally convex topological vector space $E$ is said to have the weak anti-proximinal property if every totally anti-proximinal absolutely convex subset is not rare.

Concerning the existence of bounded convex anti-proximinal sets in Banach spaces see the papers [2], [4]. We remind the reader that a subset of a topological space is said to be rare exactly when its closure has empty interior.

Conjecture 1.2 (Ricceri's Conjecture, [9]). There exists a non-complete normed space enjoying the anti-proximinal property.

In [7] it is shown, among other things, that:

- a Hausdorff locally convex topological vector space satisfies the weak anti-proximinal property if and only if it is barrelled;

- there exists a non-complete normed space enjoying the weak anti-proximinal property.

In the further sections we aim at finding the tools that allow us to improve the above results. In order to finish this introduction we will introduce the proper notation that we will make use of throughout this manuscript.

- Given a vector space $X$, the linear span or vector subspace generated by a subset $A$ of $X$ will be denoted by $\operatorname{span}(A)$.

- If $X$ is now a topological space and $A$ is a subset of $X$, then $\operatorname{int}(A)$ and $\operatorname{cl}(A)$ will denote the topological interior and the topological closure of $A$, respectively.

- Given a normed space $X$, the open unit ball of $X$ will be denoted by $\mathrm{U}_{X}$, the closed unit ball or simply the unit ball of $X$ will be denoted by $\mathrm{B}_{X}$, and the unit sphere of $X$ is $\mathrm{S}_{X}$.

\section{Quasi-absolutely convex sets}

We will introduce here a new class of convex sets called the "quasi-absolutely convex sets", which contains the absolutely convex sets as expected.

Definition 2.1. Let $X$ be a vector space. Let $A$ be a non-empty subset of $X$.

(a) We will say that $A$ is quasi-balanced if there exist $a \in A$ and $\varepsilon \in(0,1)$ such that $a-\varepsilon A \subseteq A$. 
(b) We will say that $A$ is quasi-absolutely convex if it is convex, quasibalanced, and $0 \in A$.

REMARK 2.2. Let $X$ be a vector space and consider a non-empty subset $A$ of $X$ :

(a) If $A$ is convex and $0 \in A$, then $[0,1] A \subseteq A$ and $A+A=2 A$.

(b) If $A$ is absolutely convex, then $A$ is quasi-absolutely convex. Indeed, $A$ is convex and $[-1,1] A \subseteq A$, therefore $0-\varepsilon A \subseteq A$ for all $\varepsilon \in(0,1)$.

The reader may find easy to construct quasi-absolutely convex sets which are not absolutely convex. The next theorem justifies why we consider the quasiabsolutely convex sets in lieu of the absolutely convex ones.

THEOREM 2.3. Let $X$ be a vector space and consider a quasi-absolutely convex subset $A$ of $X$. If the absolutely convex hull of $A$ is not rare, then $A$ is not rare either.

Proof. By hypothesis we may consider $a \in A$ and $\varepsilon \in(0,1)$ such that $a-\varepsilon A \subseteq A$. We will follow several steps:

- In the first place, we will prove that $a+\varepsilon \in \operatorname{co}(A \cup-A) \subseteq A+A$. Indeed, let $b, c \in A$ and $t \in[0,1]$. Notice that

$$
a+\varepsilon(t b+(1-t)(-c))=(\varepsilon t) b+(a+\varepsilon(1-t)(-c)) \in A+A
$$

in virtue of (a) in Remark 2.2.

- In the second and last place, observe that $A+A$ is not rare (in virtue of the previous point) and $A+A=2 A$ (in virtue of (1) in Remark 2.2), therefore $2 A$ is non-rare and so is $A$.

As an immediate consequence of the previous result we have the following corrollary, which constitutes the main result in this section and one of the main results in the paper.

Corollary 2.4. Let $X$ be a vector space. Then $X$ satisfies the weak antiproximinal property if and only if every totally anti-proximinal quasi-absolutely convex subset of $X$ is not rare.

Proof. - If every totally anti-proximinal quasi-absolutely convex subset of $X$ is not rare, then $X$ satisfies the weak anti-proximinal property since (2) of Remark 2.2 assures that every absolutely convex subset of $X$ is quasi-absolutely convex.

- Conversely, assume that $X$ has the weak anti-proximinal property and consider any totally anti-proximinal quasi-absolutely convex subset $A$ of $X$. We may assume without any loss of generality that $0 \in A$ in virtue of (2) of $[7$, Remark 3.1]. By applying (3) of [7, Remark 3.7] we have that the absolutely convex 
hull of $A$ is totally anti-proximinal, therefore it will be not rare by hypothesis. Finally, Theorem 2.3 allows us to deduce that $A$ is not rare either.

The reader may notice that the previous corollary constitutes a partial positive solution to Ricceri's Conjecture 1.2 in the following sense:

REMARK 2.5 (Partial Positive Solution to Ricceri's Conjecture 1.2). If every totally anti-proximinal convex set containing 0 is quasi-absolutely convex, then Ricceri's Conjecture 1.2 holds true. Indeed, if every totally anti-proximinal convex set containing 0 is quasi-absolutely convex, then the weak anti-proximinal property and the anti-proximinal property are equivalent, and according to [7, Theorem 1.3] there exists a non-complete normed space enjoying the antiproximinal property.

The previous remark arises the question whether every totally anti-proximinal convex set containing 0 is quasi-absolutely convex. In a very recent paper (see [8, Example 3.6]), the authors of this manuscript have found an example of a convex set containing 0 which is not quasi-absolutely convex. To finish this section, we will show that in finite dimensional normed spaces the bounded, convex sets containing 0 are always quasi-absolutely convex.

Theorem 2.6. Let $X$ be a finite dimensional normed space. If $A$ is a bounded convex subset of $X$ containing 0 , then $A$ is quasi-absolutely convex.

Proof. Let $Y:=\operatorname{span}(A)$. In accordance to [6, Theorem 2.1] we deduce that $\operatorname{int}_{Y}(A) \neq \emptyset$. Let $a \in A$ and $\varepsilon, \tau>0$ such that $\mathrm{B}_{Y}(a, \varepsilon) \subseteq A \subseteq \mathrm{B}_{Y}(a, \tau)$. Finally, it suffices to notice that

$$
\begin{aligned}
a-\frac{\varepsilon}{\tau+\varepsilon} A & \subseteq a-\frac{\varepsilon}{\tau+\varepsilon} \mathrm{B}_{Y}(a, \tau)=a-\frac{\varepsilon}{\tau+\varepsilon}\left(a+\tau \mathrm{B}_{Y}\right)=\frac{\tau}{\tau+\varepsilon} a-\frac{\varepsilon \tau}{\tau+\varepsilon} \mathrm{B}_{Y} \\
& =\frac{\tau}{\tau+\varepsilon}\left(a-\varepsilon \mathrm{B}_{Y}\right)=\frac{\tau}{\tau+\varepsilon} \mathrm{B}_{Y}(a, \varepsilon) \subseteq \frac{\tau}{\tau+\varepsilon} A \subseteq A
\end{aligned}
$$

if we take into consideration (a) of Remark 2.2.

\section{Intrinsic structure of totally anti-proximinal convex sets}

This section is divided into four subsections. The first one is on the possible relationship between finitely open sets and totally anti-proximinal sets; the second one is on the hereditary properties of totally anti-proximinal convex sets when intersected with proper vector subspaces; the third one is on the absorbing properties of totally anti-proximinal convex subsets; and the fourth and last one is on the semi-norms generated by totally anti-proximinal sets.

3.1. Finitely open sets. We remind the reader that the Euclidean topology on a finite dimensional vector space is the unique Hausdorff vector topology on it (which turns out to be normable). 
Definition 3.1. Let $E$ be a vector space. A subset $A$ of $E$ is said to be finitely open if for every finite dimensional vector subspace $F$ of $E$, the set $A \cap F$ is open in the Euclidean topology of $F$.

The first result in this subsection assures that the finitely open sets coincide exactly with the linearly open sets, that is, the sets composed only of internal points (see [3]).

TheOrem 3.2. Let $E$ be a vector space. A non-empty subset $A$ of $E$ is finitely open if and only if $A=\operatorname{inter}(A)$.

Proof. If $A=\operatorname{inter}(A)$, then by definition $A$ is open in the finest locally convex vector topology on $X$. Since this topology turns out to be Hausdorff, we have that the corresponding relative topology on every finite dimensional vector subspace of $E$ is indeed the Euclidean topology. As a consequence, $A$ is finitely open. Conversely, assume that $A$ is finetely open. Let $a \in A$ and consider a straight line passing through $a$. Consider the now the finite dimensional subspace $F$ generated by that straight line. By hypothesis, $A \cap F$ is open in the Euclidean topology of $F$, therefore there must exist a small interval of the straight line around $a$ entirely contained in $A \cap F$. This shows that $a \in \operatorname{inter}(A)$.

As a consequence, we find a different proof of the fact that every set composed only of internal points is totally anti-proximinal (see [7, Theorem 4.1 (1)]).

Corollary 3.3. Let $E$ be a vector space. Let $A$ be a non-empty subset of $E$ such that $A=\operatorname{inter}(A)$. Then $A$ is totally anti-proximinal.

Proof. Suppose to the contrary that $A$ is not totally anti-proximinal. There exist a norm $\|\cdot\|$ on $E$ and two elements $e \in E \backslash A$ and $a \in A$ such that $d(e, A)=\|e-a\|$. Consider the finite dimensional subspace $F:=\operatorname{span}\{e, a\}$. Observe then that $e \in F \backslash(A \cap F), a \in A \cap F$, and

$$
\|e-a\|=d(e, A) \leq d(e, A \cap F) \leq\|e-a\|,
$$

which implies among other things that $A \cap F$ is not totally anti-proximinal in $F$. By bearing in mind Theorem 3.2 we deduce that $A \cap F$ is open in the Euclidean topology of $F$. However, open subsets in the Euclidean topology of finite dimensional vector spaces are well-known to be totally anti-proximinal.

It is well known that the union of any family of finitely open sets is finitely open. The end of this subsection is aimed at showing that the totally antiproximinal sets also share this property.

Theorem 3.4. Let $X$ be a normed space. Let $\left\{A_{i}\right\}_{i \in I}$ be a family of antiproximinal subsets of $X$. If $\bigcup_{i \in I} A_{i} \neq X$, then $\bigcup_{i \in I} A_{i}$ is anti-proximinal. 
Proof. Let $x \in X \backslash \bigcup_{i \in I} A_{i}$ and $c \in \bigcup_{i \in I} A_{i}$ such that $d\left(x, \bigcup_{i \in I} A_{i}\right)=\|x-c\|$. There exists $j \in I$ such that $c \in A_{j}$. Observe now that $x \notin A_{j}$ and

$$
\|x-c\|=d\left(x, \bigcup_{i \in I} A_{i}\right) \leq d\left(x, A_{j}\right) \leq\|x-c\|,
$$

which means that $A_{j}$ is not anti-proximinal.

Corollary 3.5. Let $X$ be a vector space. Let $\left\{A_{i}\right\}_{i \in I}$ be a family of totally anti-proximinal subsets of $X$. If $\bigcup_{i \in I} A_{i} \neq X$, then $\bigcup_{i \in I} A_{i}$ is totally antiproximinal.

3.2. Inheritance of total anti-proximinality. Observe that the proof of Corollary 3.3 can be adapted to show the following.

Theorem 3.6. Let $E$ be a normed space. Let $A$ be a non-empty subset of $X$. If $A \cap F$ is anti-proximinal in $F$ for every finite dimensional subspace $F$ of $E$, then $A$ is anti-proximinal in $E$.

Proof. Suppose to the contrary that $A$ is not anti-proximinal. There exist two elements $e \in E \backslash A$ and $a \in A$ such that $d(e, A)=\|e-a\|$. Consider the finite dimensional subspace $F:=\operatorname{span}\{e, a\}$. Observe then that $e \in F \backslash(A \cap F)$, $a \in A \cap F$, and

$$
\|e-a\|=d(e, A) \leq d(e, A \cap F) \leq\|e-a\|,
$$

which implies that $A \cap F$ is not anti-proximinal in $F$.

As a corollary we obtain the version of the previous theorem for totally antiproximinal sets.

Corollary 3.7. Let $E$ be a vector space. Let $A$ be a non-empty subset of $X$. If $A \cap F$ is totally anti-proximinal in $F$ for every finite dimensional subspace $F$ of $E$, then $A$ is totally anti-proximinal in $E$.

The previous result somehow highlights the inheritance properties of totally anti-proximinal sets. By means of the remark we pretend to show that in order to accomplish the converse to the previous corollary half-lines are not suitable to use.

Remark 3.8. Consider a subset $A$ of a normed space $X$. The metric projection of $A$ is defined as

$$
\mathrm{P}_{A}: X \rightarrow \mathcal{P}(A), \quad x \mapsto \mathrm{P}_{A}(x):=\{a \in A: d(x, A)=\|x-a\|\}
$$

where $\mathcal{P}(A)$ denotes the power set of $A$. Well known properties related to the metric projections are the following. Let $x \in X \backslash A$ and $a \in \mathrm{P}_{A}(x)$ :

(a) $(a, x) \cap A=\emptyset$. 
(b) $a \in \mathrm{P}_{A}(a+t(x-a))$ for all $t \in(0,1)$.

(c) If $A$ is convex, then $A$ is a sun.

On the other hand, $A$ is said to be

- a sun provided that for every $x \in X \backslash A$ there exists a point $a \in \mathrm{P}_{A}(x)$ such that $a \in \mathrm{P}_{A}(a+t(x-a))$ for all $t>0$.

- a strict sun provided that $A$ is proximinal and for every $x \in X \backslash A$ and every $a \in \mathrm{P}_{A}(x), a \in \mathrm{P}_{A}(a+t(x-a))$ for all $t>0$.

- an $\alpha$-sun provided that for every $x \in X \backslash A$ there exists a ray $\ell$ starting from $x$ such that $d(z, A)=\|z-x\|+d(x, A)$ for every $z \in \ell$.

In general, every strict sun is a sun and every sun is an $\alpha$-sun. In [5, Chapter 12] the notion of sun is considered only for Chebyshev sets, when all the three notions above agree. For the convexity of Chebyshev sets and suns in normed spaces see, for instance, the survey paper [1].

We are particulary interested in showing that totally anti-proximinality is an hereditary property to vector subspaces at least for convex sets. So assume that $X$ is a normed space, $A$ is a convex subset of $X$, and $F$ is a vector subspace of $X$. If $A \cap F$ is not anti-proximinal in $F$, then we can find $f \in F \backslash(A \cap F)$ and $a \in \mathrm{P}_{A \cap F}(f)$. At this stage, it would be interesting to prove that $a \in \mathrm{P}_{A}(f)$ and this way $A$ is not anti-proximinal in $X$. If $a \notin \mathrm{P}_{A}(f)$, then by the previous remark we have that $a \notin \mathrm{P}_{A}(a+t(f-a))$ for any $t>0$, and so half-lines are not suitable to show that $A$ is not anti-proximinal in $X$.

3.3. Absorbing properties of totally anti-proximinal convex sets. We recall the reader that a subset $A$ of a vector space $X$ is said to be a generator system provided that its linear span is the whole of $X$, in order words, $\operatorname{span}(A)=$ $X$. In [6, Lemma 2.4] it is shown that in a topological vector space an absolutely convex subset is absorbing if and only if it is a generator system. A slighter version of this is presented in the following sense: if a convex set contains 0 and is a generator system, then it "absorbs" all the "positive" vectors (relative to a given Hamel basis). In an more precise language:

Lemma 3.9. Let $X$ be a real vector space. Let $A$ be a convex subset of $X$ containing 0 which is also a generator system of $X$. Let $\left\{e_{i}: i \in I\right\}$ be a Hamel basis of $X$ contained in $A$. Let $x \in X \backslash\{0\}$ such that $x=\lambda_{1} e_{i_{1}}+\ldots+\lambda_{p} e_{i_{p}}$ with $\min \left\{\lambda_{i}: i \in\{1, \ldots, p\}\right\}>0$. Then there exists $\lambda \geq 0$ such that $\gamma x \in A$ for all $0 \leq \gamma \leq \lambda$.

Proof. In virtue of Remark 2.2 it suffices to consider $\lambda=1 /\left(\lambda_{1}+\ldots+\lambda_{p}\right)$

REMARK 3.10. Let $X$ be a real vector space. Let $A$ be a convex subset of $X$ containing 0 which is also a generator system of $X$. Let $\left\{e_{i}: i \in I\right\}$ be a Hamel 
basis of $X$ contained in $A$. As indicated in the statement of the previous lemma, the convex cone of "positive" vectors of $X$ relative to $\left\{e_{i}: i \in I\right\}$ is given by

$$
P:=\left\{\lambda_{1} e_{i_{1}}+\ldots+\lambda_{p} e_{i_{p}}: \lambda_{i_{j}} \geq 0 \text { for } 1 \leq j \leq p\right\} .
$$

The previous lemma assures that

$$
P=\bigcup_{n \in \mathbb{N}} n(A \cap P)
$$

which means, among other things, that if $X$ is endowed with a vector topology that makes $P$ a Baire space, then $A \cap P$ is not rare in $P$. Nevertheless, in infinite dimensional topological vector spaces the convex cone $P$ has empty interior as shown in the next result.

THEOREM 3.11. Let $X$ be a real topological vector space and consider a Hamel basis $\left\{e_{i}: i \in I\right\}$ for $X$. Consider the convex cone of "positive" vectors of $X$ relative to $\left\{e_{i}: i \in I\right\}$, that is, $P:=\left\{\lambda_{1} e_{i_{1}}+\ldots+\lambda_{p} e_{i_{p}}: \lambda_{i_{j}} \geq 0\right.$ for $\left.1 \leq j \leq p\right\}$. The following conditions are equivalent:

(a) $X$ is finite dimensional.

(b) $\operatorname{inter}(P) \neq \emptyset$.

Proof. (a) $\Rightarrow$ (b). If $X$ is finite dimensional, then it is enough to check that

$$
\operatorname{inter}(P)=\left\{\lambda_{1} e_{1}+\ldots+\lambda_{p} e_{p}: \lambda_{j}>0 \text { for } 1 \leq j \leq p\right\} .
$$

(b) $\Rightarrow$ (a). Assume that $X$ is infinite dimensional. Let $\lambda_{1} e_{i_{1}}+\ldots+\lambda_{p} e_{i_{p}} \in P$ with $\lambda_{i_{j}} \geq 0$ for $1 \leq j \leq p$. Since $I$ is infinite, we can find $i_{0} \in I \backslash\left\{i_{1}, \ldots, i_{p}\right\}$. Notice now that if $t<0$ then $t e_{i_{0}}+(1-t)\left(\lambda_{1} e_{i_{1}}+\ldots+\lambda_{p} e_{i_{p}}\right) \notin P$.

In virtue of $[7$, Theorem 2.2] we deduce that the totally anti-proximinal convex subsets that contain 0 "absorbs" all the "positive" vectors in the sense of Lemma 3.9. The following lemma, which concludes this subsection and whose proof we omit for being obvious, provides us with a possible way to show that totally anti-proximinal convex subsets that contain 0 are actually absorbing.

Lemma 3.12. Let $X$ be a vector space. Let $A$ be a subset of $X$ containing 0. The following conditions are equivalent:

(a) $A$ is absorbing.

(b) $0 \in \operatorname{inter}(A)$.

3.4. Norms generated by totally anti-proximinal convex sets. In this subsection we will consider totally anti-proximinal convex sets which are "linearly bounded" and study the properties of the norm generated by their absolutely convex hull. We will begin by the definition of "linearly bounded". 
Definition 3.13. Let $X$ be a vector space. A non-empty subset $A$ of $X$ is said to be linearly bounded exactly when $A$ does not contain any semi-straight line, or equivalently, all the maximal segments of $A$ are bounded.

The reader may notice that an absolutely convex set is linearly bounded if and only if does not contain vector subspaces.

REMARK 3.14. Let $X$ be a normed space. If $x \in X \backslash \mathrm{B}_{X}$, then $d\left(x, \mathrm{~B}_{X}\right)=$ $\|x\|-1$. Indeed,

$$
d\left(x, \mathrm{~B}_{X}\right) \leq\left\|x-\frac{x}{\|x\|}\right\|=\|x\|-1,
$$

and if $y \in \mathrm{B}_{X}$, then

$$
\|x\|-1 \leq\|x\|-\|y\| \leq|\|x\|-\|y\|| \leq\|x-y\| .
$$

Lemma 3.15. Let $E$ be a vector space. Let $A$ be a non-empty subset of $E$. If $A$ is totally anti-proximinal and is contained in the closed unit ball of a norm on $X$, then $A$ is actually contained in the open unit ball of that norm.

Proof. Let $\|\cdot\|$ be any norm on $X$ whose closed unit ball $\mathrm{B}_{\|\cdot\|}$ contains $A$. Suppose to the contrary that there exists $a \in A \cap \mathrm{S}_{\|} \cdot \|$. By applying Remark 3.14 we have that

$$
1=\|2 a-a\| \geq d(2 a, A) \geq d\left(2 a, \mathrm{~B}_{\|\cdot\|}\right)=\|2 a\|-1=1,
$$

which means that $A$ is not totally anti-proximinal.

We will finish this subsection with the following result.

TheOREM 3.16. Let $X$ be a vector space. Suppose that $A$ is a linearly bounded, totally anti-proximinal convex subset of $X$. Then the absolutely convex hull of A coincides with the open unit ball of the norm that it generates and hence it is finitely open.

Proof. It is well known that in this case and since $A$ is convex, the absolutely convex hull of $A$ is given by $\operatorname{co}(A \cup-A)$. In the first place, we have that $\operatorname{span}(A)=$ $X$ in virtue of $[7$, Theorem 3.2]. As a consequence, $\operatorname{co}(A \cup-A)$ is also a generator system of $X$. Now we apply [6, Lemma 2.4] to conclude that $\operatorname{co}(A \cup-A)$ is absorbing. Denote by $\|\cdot\|$ the norm generated by the absolutely convex hull of $A$. Since

$$
A \subseteq \operatorname{co}(A \cup-A) \subseteq \mathrm{B}_{\|\cdot\|},
$$

by applying Lemma 3.15 we deduce that $A \subseteq \mathrm{U}_{\|\cdot\|}$, which automatically implies in virtue of the triangular inequality that $\operatorname{co}(A \cup-A)=\mathrm{U}_{\|\cdot\| \cdot}$

The reader may note that the previous result is an improvement of (3) of $[7$, Remark 3.7]. 


\section{Comparison of norms and barrelledness}

In this section we provide a characterization of barrelledness in terms of comparison of norms.

REMARK 4.1. Let $X$ be a vector space and consider two norms $|\cdot|$ and $\|\cdot\|$ on $X$. It is well known that the following four assertions are equivalent:

(a) There exists $K>0$ such that $|\cdot| \leq K\|\cdot\|$.

(b) The topology induced by $|\cdot|$ is contained in the topology induced by $\|\cdot\|$.

(c) The unit ball of $\|\cdot\|, \mathrm{B}_{\|\cdot\|}$, is bounded in $(X,|\cdot|)$.

(d) $\mathrm{B}_{|\cdot|}$ has non-empty interior in $(X,\|\cdot\|)$.

In particular, any of the conditions above implies that $\mathrm{B}_{|\cdot|}$ is closed in $(X,\|\cdot\|)$. We will show now that this last assertion is equivalent to all four points above only when $X$ is barrelled.

REMARK 4.2. Let $X$ be a vector space. Let $A$ be a non-empty subset of $X$. Note that if $A$ is absorbing, then the Minkowski functional on $A, \phi_{A}$, is well defined. Recall that $\phi_{A}(x):=\inf \{\lambda>0: x \in \lambda A\}$ for all $x \in X$. If, in addition, $A$ is absolutely convex, then $\phi_{A}$ is a semi-norm on $X$ which verifies that $\mathrm{U}_{\phi_{A}} \subseteq A \subseteq \mathrm{B}_{\phi_{A}}$. Finally, if, on top of everything else, $A$ is linearly bounded, then $\phi_{A}$ is a norm on $X$.

Lemma 4.3. Let $X$ be a topological vector space. Let $A$ be a barrel of $X$ and denote by $|\cdot|$ the semi-norm on $X$ given by the Minkowski functional of $A$. Then $A=\mathrm{B}_{|\cdot|}$.

Proof. By Remark 4.2 we have that $\mathrm{U}_{|\cdot|} \subseteq A \subseteq \mathrm{B}_{|\cdot|}$. Let $x \in \mathrm{B}_{|\cdot|}$ and consider any $u \in \mathrm{U}_{|\cdot|}$. It is well known that $[u, x) \subset \mathrm{U}_{|\cdot|}$, therefore $(u / n+(1-$ $1 / n) x)_{n \in \mathbb{N}}$ is a sequence in $A$ which converges to $x$ in the original vector topology of $X$. Since $A$ is closed in that vector topology, we deduce that $x \in A$.

We will conclude this manuscript by providing a characterization of barrelledness in terms of comparison of norms.

TheOREM 4.4. Let $X$ be a normed space with norm $\|\cdot\|$. The following conditions are equivalent:

(a) $X$ is barrelled.

(b) If $|\cdot|$ is a norm on $X$ whose unit ball is closed in the topology induced by $\|\cdot\|$, then there exists $K>0$ such that $|\cdot| \leq K\|\cdot\|$.

Proof. (a) $\Rightarrow$ (b). Assume that $X$ is barrelled and let $|\cdot|$ be a norm on $X$ whose unit ball, $B_{|\cdot|}$, is closed in the topology induced by $\|\cdot\|$. Notice then that $\mathrm{B}_{|\cdot|}$ is a barrel of $X$ and thus it has non-empty interior. It is sufficient now to apply Remark 4.1. 
(b) $\Rightarrow$ (a). Let $A$ be any barrel of $X$. Notice that we may assume without any loss of generality that $A$ is bounded since we can intersect it with the unit ball of $X$. So, let us suppose that $A$ is bounded. Denote by $|\cdot|$ the norm on $X$ given by the Minkowski functional of $A$. Since $A$ is closed in $(X,\|\cdot\|)$, by Lemma 4.3 we have that $A=\mathrm{B}_{|\cdot|}$ and hence $\mathrm{B}_{|\cdot|}$ is closed in $(X\|\cdot\|)$. By hypothesis, there exists $K>0$ such that $|\cdot| \leq K\|\cdot\|$, which means that $A$ is a neighbourhood of 0 in $(X\|\cdot\|)$ by bearing in mind Remark 4.1.

\section{REFERENCES}

[1] V.S. BalaganskiJ and L.P. Vlasov, The problem of convexity of Chebyshev sets, Russ. Math. Surv. 51 (1996), No. 6, 1127-1190; transl. from Usp. Mat. Nauk 51 (1996), No. 6, 125-188; errata ibid. 52 (1997), No. 1, 237.

[2] J.M. Borwein, M. Jiménez-Sevilla And J.P. Moreno, Antiproximinal norms in Banach spaces, J. Approximation Theory 114 (2002), no. 1, 57-69.

[3] N. Bourbaki, Topological vector spaces, Chapters 1-5, transl. from the French by H.G. Eggleston and S. Madan, Elements of Mathematics (Berlin), Springer-Verlag, Berlin, 1987.

[4] S. Cobzas, Antiproximinal sets in Banach spaces, Acta Univ. Carol., Math. Phys. 40 (1999), no. 2, 43-52.

[5] F. Deutsch, Best approximation in inner product spaces, CMS Books in Mathematics/Ouvrages de Mathématiques de la SMC. 7 NY, Springer, New York, 2001.

[6] F.J. GARCíA-PACheCO, Non-continuous linear functionals on topological vector spaces, Banach J. Math. Anal. 2 (2008), no. 1, 11-15.

[7] _ An approach to a Ricceri's conjecture, Top. Appl. 159 (2012), 3307-3313.

[8] F.J. García-Pacheco and J.R. Hill, Advances on Ricceri's most famous conjecture, Filomat 29 (2015), no. 4, 829-838.

[9] B. RICCERI, Topological problems in nonlinear and functional analysis, Open Problems in Topology II (E. Pearl, ed.), Elsevier, 2007, 585-593.

Manuscript received December 31, 2012

Francisco J. García-Pacheco

Department of Mathematical Sciences

University of Cadiz

Puerto Real, 11510, SPAIN

E-mail address: garcia.pacheco@uca.es

Justin R. HiLL

Department of Mathematics and Physics

Texas A\&M University

Central Texas, Killeen, TX, 76549, USA

E-mail address: justin.hill@ct.tamus.edu

TMNA : Volume $46-2015-\mathrm{N}^{\mathrm{O}} 1$ 